\title{
Comparative transcriptome analysis provides insights into seed germination in herbicide resistance Polypogon fugax
}

Xiaoyue Yu ( $\nabla$ yuxiaoyue@caas.cn )

China national rice research institute

\section{Wei Tang}

China National Rice Research Institute

\section{Yongjie Yang}

China National Rice Research Institute

Jianping Zhang

China National Rice Research Institute

\section{Yongliang Lu}

China National Rice Research Institute

\section{Research article}

Keywords: seed germination, Polypogan fugax, herbicide resistance, transcriptome

Posted Date: June 2nd, 2020

DOl: https://doi.org/10.21203/rs.3.rs-24962/v1

License: (c) (1) This work is licensed under a Creative Commons Attribution 4.0 International License. Read Full License 


\section{Abstract \\ Background}

Herbicide resistant mutations are predicted to exhibit fitness cost under herbicide-free conditions. Asia minor bluegrass (Polypogon fugax) is a common weed species in the winter crops. Our previous study established a $P$. fugax accession (LR) resistant to acetyl-CoA carboxylase (ACCase) inhibiting herbicides. Besides, LR also exhibited fitness cost, like lower germination, relative to the sensitive plants (LS). But little is known about the gene expression profile of seed germination process in herbicide resistance P.fugax.

\section{Results}

The maximum germination (gMAX) of LS reached $94 \%$, while that of LR was $52 \%$. Exogenous gibberellin and abscisic acid synthesis inhibitor fluridone could break the dormancy of LR, increasing gMAX to $78 \%$ and $94 \%$, respectively. A comparative transcriptome was conducted to analyze the genes expression profile of LS and LR at two germination time points. A total of 11,856 and 23,123 differentially expressed genes (DEGs) were identified in LS and LR at two time points. Most of DEGs were involved in lipid metabolism, carbohydrate metabolism, amino acid metabolism and secondary metabolites biosynthesis. Four genes related to GA biosynthesis and signal transduction showed higher expression in LS and five genes related to ABA biosynthesis and signal transduction showed higher expression in LR during germination. Twenty-four genes involved in glycolysis, TCA cycle, pentose phosphate pathway and fatty acid metabolism had higher expression levels in LS than LR during germination.

\section{Conclusions}

Our results indicated that GA biosynthesis and transduction, carbohydrate and fatty acid metabolism was more actively expressed in LS plants, resulting in stronger germination ability of LS relative to LR. The highly expressed genes in ABA biosynthesis and transduction contribute to maintain the seed dormancy of LR plants. This study provided new insight into transcriptional changes and interaction in seed germination process of $P$. fugax, and compared the differential expression profile between the herbicides resistant and sensitive plants during seed germination.

\section{Background}

Herbicide resistant mutations were considered as pre-existed or arise spontaneously with very low rate within weeds populations [1-3]. With frequent herbicide application, herbicide resistant mutations are rapidly selected and enriched in weed populations [4]. Without herbicide stress, herbicide resistant mutations are predict to exhibit an adaptation cost (fitness cost) due to the impaired enzyme ability or altered feedback inhibition resulting in insufficient or excessive product biosynthesis [5-7]. Many 
herbicide resistant weeds exhibited altered phenotypes, including seed germination ability, growth cycle, biomass production, competitive ability and reproduction $[8,9]$. For instance, the acetolactate synthase (ALS) and ACCase herbicides multiple-resistance Lolium rigidum biotypes showed lower vigor and competitive ability against wheat than that of the sensitive biotype [10]. The Alopecurus myosuroides ACCase gene mutant showed a reduction of biomass, height and seed production [11]. Two Hordeum glaucum ACCase gene mutants exhibited lower growth rate, biomass and seed production [12].

Asia minor bluegrass (Polypogon fugax) is a common winter weed species distributed across China and other Asian countries. This annual grass has an extended period of emergence from early November to late December. Its life cycle is highly close to several winter crops, including wheat (Triticum aestivum L.), rapeseed (Brassica napus L.) and some vegetables $[13,14]$. $P$. fugax is a competitive weed, especially in moist soil and has become an increasing problem in wheat or rapeseed fields in rotation with rice [15]. In our previous study, a $P$. fugax accession (LR) collected from Sichuan province in China was highly resistant to ACCase inhibiting herbicides. The resistance mechanism was conferred by an lle-2041-Asn substitution in ACCase gene [16]. In addition, this herbicide resistance plants exhibited fitness cost, including lower germination and emergence potential, earlier flowering and tiller and panicle emergence and seed shedding, relaively to the sensitive plants $[17,18]$. A transcriptome study of the P.fugax resistant and sensitive plants were performed at the flowering stage, which provide a genomic resource for understanding the molecular basis of early flowering. The study identified twelve genes exhibited different expression pattern in herbicide resistant plants and sensitive plants [19]. But the molecular basis of lower germination ability in the herbicide resistance plants was still unclear. Whether the altered phenotypes were a consequence of pleiotropic effects of resistance mutations was difficult to figure out. The fitness cost occurred in herbicide resistance mutations are not universal, which depends on the particular mutation[11, 20-22], genetic background [23], and environmental conditions [24, 25]. Thus, the sensitive plants used in this study were collected from the same habitat of resistant plants to minimize the genetic background variance. The resistance and sensitive plants reproduced for three generations under the same environmental condition to minimize the influence of environmental conditions. These limitations allowed us to focus on the effect of ACCase mutation on fitness cost.

Seed germination and dormancy are important components of plant fitness with complex regulation network, including energy production, protein metabolism, phytohormone control and transcription factors regulation. With the development of genomics, transcriptomic analysis was one of the most effective and economic approaches to investigate the difference of germination process between the tested samples. A transcriptome analysis of two maize inbred lines with different germination ability identified abundant genes associated with plant hormones, nutrient reservoir, amino acid metabolism, and ribsome in driving seed germination. Genes related to plant hormones and amino acid metabolism showed higher expression level in the maize with high germination ability [26]. A comparative transcriptome analysis between two sheepgrass (Leymus chinensis) germplasm with various germination percentages at three developing stages indicated that genes involved in starch and sucrose metabolism, phenylpropanoid biosynthesis, plant hormone signal transduction, amino sugar and nucleotide sugar metabolism, and photosynthesis were significantly changed [27]. The aim of this study 
is to investigate the differential expression profile of seed germination process in the herbicide resistant and sensitive plants, and try to figure out the relevant biological pathways or function genes contribute to germination delay in herbicide resistant plants.

\section{Results And Discussion}

\section{Characterization of seed germination in LS and LR}

The maximum germination (gMAX) of LR was about $52 \%$ while that of LS reached $94 \%$ (Fig 1a), consistent with the previous study results [17], suggesting that the low germination character was steadily inherited in LR. According to the germination curve, the rapid growth period was from $3 \mathrm{~d}$ to $8 \mathrm{~d}$ after incubated (DAI). Seed germination is a process with complex physiological, biochemical and molecular biological basis [28]. With the absorption of water, the storage macromolecules such as starch, proteins and lipids were decomposed into small and soluble molecules that can be easily utilized and transformed to support the germination [29]. The content of soluble sugar in the 0 DAl seeds of LS was higher than LR. After incubation, the soluble sugar content deceased and then dramatically increased at 6 DAI in LS and LR seeds. The soluble sugar content of LR seeds was always lower than that of LS seeds until 8 DAl (Fig. 1b). Similarly, the content of soluble protein showed down first and then up, and the content in LR was lower than that in LS during the germination process (Fig. 1c). These results indicated that more soluble sugar and protein could directly participate carbon oxidation and enzyme catalysis to supply energy in LS in comparison with LR during germination. Combined with the dynamic change of germination rate and soluble sugar and protein content, we chose 3 DAl and 6 DAl seeds for transcriptome analysis to compare the difference of germination process between LS and LR.

\section{Hormones regulate the seed germination of LS and LR}

Gibberellin (GA) and abscisic acid (ABA) antagonistically regulate the transition of germination and dormancy, that GA promote germination and ABA promote dormancy [30] To investigate whether the germination delay of LR was related to the hormonal regulation, the seeds of LS and LR were treated with exogenous $G A$ and $A B A$ and their synthesis inhibitors, fluridone $(F L)$ and paclobutrazol (PA), to observed the gMAX values (Fig. 1d). The application of GA significantly evaluated the gMAX of LR from $52 \%$ to $78 \%$, suggesting that exogenous GA could promote the germination of LR. The application of ABA had no significant effect on the gMAX of LS but decrease that of LR to $17 \%$, suggesting that the inhibitory by $A B A$ was stronger in LR than LS. PA and FL inhibits the GA and ABA biosynthesis had been proved in many plant species [31]. The application of PA decreased the gMAX of LS to $73 \%$ and that of LR decreased to $25 \%$, suggesting that the GA biosynthesis in LR was more strongly inhibited by PA. The application of FL dramatically increased the gMAX of LR to $94 \%$, suggesting that the dormancy of $L R$ completely removed when ABA biosynthesis was inhibited.

\section{de novo assembly of P.fugx reference transcriptome}


Two $P$. fugax accessions LS and LR were incubated for $3 \mathrm{~d}$ and $6 \mathrm{~d}$, respectively, and three biological replicates were designed in each accession at two time points. Thus twelve samples were used to conduct the RNA-seq analysis. The number of total clean reads of each samples were shown in Table 1. A total of 476,220 transcripts and 149,330 genes were de novo assembled, and the distribution of length interval was shown in Fig. 2a.

The number of annotated unigenes of each database was show in Table 2. Basically, a total of 99,209 genes $(66.43 \%)$ were at least annotated to one database, suggesting that the de novo transcriptome had relatively complete gene function information. Among them, 67,183 genes $(44.98 \%)$ were annotated to NR database, and established that P.fugax were highly similar to Aegilops tauschii subsp. tauschii (30.4\%), Brachypodium distachyon (L.) P. Beauv. (16.5\%), Hordeum vulgare subsp. vulgare (8.8\%), Triticum urartu (5.6\%), and Oryza sativa Japonica Group (4.4\%) (Fig. 2b). A total of 20,042 genes $(13.42 \%)$ were annotated into 25 classifications of KOG database (Additional file 2). Among them, the highest number of categories were "posttranslational modification, protein turnover, chaperones" (2,701, $13.48 \%)$, "general function prediction only" $(2,627,13.11 \%)$ and "translation, ribosomal structure and biogenesis" (2,321, 11.58\%) (Fig. 2c). Moreover, there were 61,384 genes (41.1\%) annotated to GO database, 22,834 unigenes (15.29\%) annotated to KEGG database, $68,540(45.89 \%)$ to $\mathrm{Nt}$ database, and $52,642(35.25 \%)$ to SwissProt database (Additional file 3, 4).

\section{Identification of differentially expressed genes (DEGs)}

To determine the molecular basis involved in the germination process of $L S$ and $L R$, two comparison settings, LS_6d vs LS_3d and LR_6d vs LR_3d, were analyzed. The biological samples had good reproducibility due to the high correlation values (Fig. $3 \mathrm{c}$ ). The distribution of the DEGs in LS and LR were directly exhibited in volcano plots (Fig. 3a and $3 b$ ). A total of 11,856 DEGs were identified and 8,936 were up-regulated and 2,920 were down-regulated in the LS comparison setting. A total of 23,123 DEGs were identified and 20,055 were up-regulated and 3,068 were down-regulated in the LR comparison setting. A venn diagram showed that 7,165 up-regulated DEGs and 1,167 down-regulated DEGs were common in LS and LR comparison settings (Fig. 3d).

\section{GO and KEGG enrichment of DEGs}

To understand the difference in biological function and processes related to germination between LS and LR from 3 DAl to 6 DAl, all the DEGs were enriched to GO and KEGG database. The up-regulated DEGs in LS were enriched to $109 \mathrm{GO}$ terms and the down-regulated DEGs were enriched to $9 \mathrm{GO}$ terms (Additional file 5). The up-regulated DEGs in LR were enriched to $224 \mathrm{GO}$ terms and the down-regulated DEGs were enriched to $40 \mathrm{GO}$ terms (Additional file 6). The up-regulated DEGs in LS and LR were highly enriched in "metabolic process", "single-organism process", and "catalytic activity". With regard to the down-regulated DEGs, the enriched GO terms were much less in LS than LR. These results indicated that most of the upregulated DEGs in both LS and LR had similar biological function. All the up-regulated DEGs in LS and LR were enriched to KEGG database and the top 20 enriched pathways were shown in Fig. $4 \mathrm{a}$ and $4 \mathrm{~b}$. The majority of up-regulated DEGs were related to lipid metabolism, carbohydrate metabolism, amino acid 
metabolism and secondary metabolites biosynthesis in LS and LR seed during germination. Here, we selected some pathways to investigate the difference of regulation network in LS and LR (Table 3).

\section{DEGs related to hormones biosynthesis and signal transduction}

Seeds germination and dormancy processes are regulated by diverse endogenous hormones. According to the results of exogenous hormones regulation, GA and ABA affected the seed germination of LS and LR at different levels. The genes (Cluster-37472.24032 and Cluster-37472.23672) coding two ent-kaurene synthases (KS) and gene (Cluster-37472.70387) encoding gibberellin 3beta-hydroxylase (GA3ox) were upregulated in both LS and LR comparison settings, and the increasing was much higher in LS than that in LR. The first step in GA biosynthetic pathway is transformed geranylgeranyl pyrophosphate to entkaurene catalyzed by ent-kaurene synthetases. The $K S$ deficient mutants of Arabidopsis showed strong seed dormancy and recovered germinate with exogenous GA treatment [32, 33]. GA3ox catalyzes the final biosynthetic step to produce bioactive GAs. Two Arabidopsis genes, $G A 4$ and $G A 4 H$, encoding GA3ox were expressed during seed germination [34]. These GA biosynthesis related genes with higher expression in LS suggested that GA biosynthesis was more active in LS than LR. Consistent with this result, the inhibition of GA biosynthesis by PA was more effective in LR than LS. The gene (Cluster-51396.0) encoding phytochrome-interacting factor 4 (PIF4) was down-regulated in LS but up-regulated in LR during germination. PIF4 regulated the gibberellin-signaling pathway via DELLA proteins, which blocked the GA signal pathway and resulting in germination delay [35]. Thus, the increased expression of PIF4 might repress seed germination via inhibiting the GA signal transduction in LR.

ABA is the major hormone that inducing seed dormancy. The gene (Cluster-37472.23110) coding 9-cisepoxycarotenoid dioxygenase (NCED) was down-regulated in LS but up-regulated in LR during germination. The expression of gene (Cluster-37472.23992) coding phytoene synthase (PSY) and gene (Cluster-37472.76697) coding lycopene epsilon-cyclase (LcyE) were both elevated in LS and LR during germination, and the increasing was higher in LR than LS. The cleavage of 9-cis-epoxycarotenoids catalyzed by NCED is the key regulatory step of ABA biosynthesis. The Arabidopsis mutants of NCED genes, Atnced6 and Atnced9, reduced ABA content level in seeds [36]. PSY is the limiting step of carotenoids synthesis, the upstream of ABA biosynthesis. The PSY gene overexpressed Arabidopsis mutant exhibited delayed germination, and the degree of delay was positively associated with the increased levels of carotenoids and ABA [37]. The gene (Cluster-37472.6593) encoding serine/threonineprotein kinase SRK2 (SNRK2) and gene (Cluster-48355.0) coding ABA responsive element binding factor (ABF) were up-regulated in LS and LR, and increasing were higher in LR than LS. It has established in rice and Arabidopsis that SNRK2 is activated by ABA and phosphorylate ABF, which is important for the activation of ABA signal transduction [38,39]. Taken together, these genes associated with ABA biosynthesis and signal transduction pathways exhibited higher expression level in LR than LS, which might explain why the LR seeds were more sensitive to $A B A$ and FL could restore the germination ability of LR.

\section{DEGs related to fatty acid metabolism}


ACCase catalyzes the carboxylation of acetyl-CoA to malonyl-CoA, acting as the initial step of fatty acid biosynthesis [40, 41]. ACCase has three subunits: biotin carboxylase carrier protein, biotin carboxylase, and carboxyltransferase domains [42]. Molecular and biochemical studies have proved that the carboxyltransferase domain is the target site of ACCase-inhibiting herbicides [43]. LR plants had an amino acid substitution in carboxyltransferase domain that caused the resistance to ACCase-inhibiting herbicide [16]. Unfortunately, the transcriptome data did not identify any DEGs annotated as ACCase carboxyltransferase domain in LS or LR. But gene (Cluster-39490.0) coding ACCase biotin carboxylase (ACACA) was identified to up-regulated with the higher increasing level in LS than LR. ACCase is the ratelimiting enzyme in fatty acid metabolism, the different regulation level between LS and LR might influence the downstream reactions as well. The gene (Cluster-31226.0) coding 3-oxoacyl-[acyl-carrier protein] reductase (FabG), gene (Cluster-37472.4287) coding long-chain acyl-CoA synthetase (ACSL) showed higher regulation level in LS compared with LR during germination. These enzymes were respectively involved in the elongation cycle and termination of fatty acid synthesis. Besides, the genes involved in fatty acid degradation were also up-regulated higher in LS than LR, such as the gene (Cluster33548.0) coding acetyl-CoA acyltransferase 1 (ACAA1), gene (Cluster-37472.85335) coding acyl-CoA dehydrogenase (ACADM), gene (Cluster-30044.0) coding acetyl-CoA C-acetyltransferase (ACAT). The fatty acid forms triacylglycerol (TAG) that degrades to provide carbon and energy during germination and early seedling growth. Some ACCase herbicide resistant weeds also showed higher level of dormancy, absence of germination in dark conditions, and delayed seed germination [44-46]. We assumed that the mutated ACCase might alter normal fatty acid metabolism and impact the germination.

\section{DEGs related to carbohydrate metabolism}

Acetyl-CoA, acts as the precursor of fatty acid biosynthesis, is also the product of glycolysis and TCA. According to the dynamic change of soluble sugar and protein content, more soluble sugar and protein could directly participate carbon oxidation and enzyme catalysis in LS than LR during germination. Thus, the genes involved in carbohydrate metabolism were selected to compare the differential expression pattern. The gene (Cluster-37472.6545) coding glucose-6-phosphate isomerase (GPI) 『gene (Cluster37472.71503) coding fructose-bisphosphate aldolase (FBA), gene (Cluster-35171.2) coding phosphoenolpyruvate carboxykinase (PCKA), gene (Cluster-40718.0) coding pyruvate dehydrogenase E1 component alpha subunit (PDHA), genes (Cluster-37472.39187, Cluster-37472.47005) coding alcohol dehydrogenase $(A D H)$, were up-regulated in LS and LR comparison settings, and the increasing levels were higher in LS than LR. All of these genes were involved in glycolysis, which produces energy by utilizing endosaccharides [47]. PCKA catabolizes the storage lipid and protein to produce soluble sugar via glycolysis. The PCKA deficient mutants of Arabidopsis and tomato were observed growth suppression of germinated seedlings [48].

The genes (Cluster-37472.85108, Cluster-15133.3) coding malate dehydrogenase (MDH), gene (Cluster37472.21027) coding citrate synthase (CS), gene (Cluster-33485.0) coding 2-oxoglutarate dehydrogenase E1 component (OGDH), genes (Cluster-38121.0, Cluster-34010.0) coding succinyl-CoA synthetase alpha subunit and beta subunit (LSC1 and LSC2), genes (Cluster-37472.82495, Cluster-39676.0) coding 
succinate dehydrogenase (ubiquinone) iron-sulfur subunit (SDHB), and gene (Cluster-41938.0) coding pyruvate carboxylase (PYC) were up-regulated in LS and LR at 6 DAl compared with 3 DAl, and the increasing levels were higher in LS than LR. All of these genes were involved in TCA cycle, which produced high-energy phosphate compounds as the main source of cellular energy [49]. MDH catalyze the interconversion of malate and oxaloacetate in TCA cycle. The Arabidopsis $\mathrm{MDH}$ gene mutants showed slowly germination rate, higher content of free amino acids, different sugar levels, and lower content of 2-oxoglutarate [50]. The seeds of the CS deficient Arabidopsis were dormant and did not metabolize triacyglycerol [51].

The gene (Cluster-37472.72264) coding 6-phosphogluconate dehydrogenase (PGD), gene (Cluster37472.83492) coding transketolase (TKT), gene (Cluster-37472.237) coding gluconokinase (gntK) were highly up-regulated in LS compared with LR during germination. Pentose phosphate pathway takes substrate from glycolysis and feeds its products back into glycolysis, which is important in determining the flux through glycolysis [52].

One of the major changes during germination is a rapid increase in respiration, which involves glycolysis, oxidative pentose phosphate pathway, TCA cycle and oxidative phosphorylation. All of the DEGs mentioned above were related to TCA cycle, glycolysis or pentose phosphate pathway that producing energy during seed germination. These genes were up-regulated in LS and LR during germination and had higher expression in LS, indicating that more energy were supplied to promote germination in LS in comparison with LR.

\section{Validation of DEGs by qRT-PCR}

A total of sixteen DEGs were randomly selected to verify the accuracy and reproducibility of the transcriptome results by qRT-PCR (Fig. 5). The correlation between transcriptome results (FC) and qRTPCR results $\left(2^{-\Delta \Delta C t}\right)$ were calculated using $\log _{2}$ fold variation measurements to produce a scatter plot. The results showed that the expression profiles of these DEGs were consistent with the transcriptome results, with relative $R^{2}=0.8751$ and 0.7376 in $L S$ and $L R$, respectively.

\section{Conclusion}

In this study, we investigated the expression profile during seed germination in two P.fugax accessions at two time points. GA and FL promote the germination of LR plants, while ABA and PA showed greater inhibiting effect on germination of LR plants. Consistent with this, the transcriptome results identified four GA signal related genes with higher expression in LS and five ABA signal related genes with higher expression in LR during germination. LS showed higher content of soluble sugar and protein than LR during germination. The expression of the genes related to carbohydrate metabolism and fatty acid metabolism were highly increased in LS than LR, suggesting that more energy was supplied during seed germination in LS (Fig. 6). This study provided novel insight into the gene expression profile of seed germination in $P$. fugax and identified abundant function genes involved in germination delay in herbicide 
resistant plants. It should stress that this study could not summarize the effect of herbicide resistance mutation on seed germination. Because the differential expression profile of seed germination was described only in two accessions. The pathways or function genes identified in this study should be verified in other resistant plants with germination delay.

\section{Methods}

\section{Plant materials and growth condition}

The P.fugax accessions were originally collected in 2012 in or nearby a grower's field in Qingshen County, Sichuan Province, China, and identified to be resistant and sensitive to ACCase inhibiting herbicides, respectively. No specific permission is required for the $P$. fugax accessions collection, and the collected location are not privately owned or natural protected areas. The plant materials were identified by Prof. Xiaogang Zhou from Sichuan Academy of Agriculture Sciences. The voucher specimens were deposited at State Key Laboratory of Rice Biology, China National Rice Research Institute. The F2 seeds collected from the resistant plants (LR) had lower germination rate compared with the sensitive plants (LS) under various environment conditions [17]. The seeds used in this study were F3 seeds collected from the F2 plants of LR and LS that separately cultivated in December 2018 screen house (an $8 \mathrm{~m}$ by $20 \mathrm{~m}$ chamber framed with 2-cm iron mesh and covered overhead with a transparent plastic cover to prevent rain damage) at the China National Rice Research Institute (CNRRI; $\left.30.04^{\circ} \mathrm{N}, 119.55^{\circ} \mathrm{E}\right)$, Hangzhou, Zhejiang Province, China. The seeds were collected in Match 2019 and dried at $35^{\circ} \mathrm{C}$ for 7 days and stored at $4{ }^{\circ} \mathrm{C}$ under darkness for six months until use. The seed viability were tested by bromothymol blue and reached almost $100 \%$.

\section{Dynamic changes in germination percentage, soluble sugar and protein content}

The peeled seeds of LS and LR were surface sterilized with 70\% ethanol for 1 min and $10 \%$ sodium hypochlorite for $10 \mathrm{~min}$ and then washed with sterile water for three times. Sterile seeds placed on the 9 $\mathrm{cm}$ petri dishes with one layer of filter paper that moistened with sterile water. The seeds were incubated at $20 / 10{ }^{\circ} \mathrm{C}$ day/night cycles with a $12 \mathrm{~h}$ photoperiod in the growth chamber. The seed was considered as germinated when the radicle emergence to the seed length. The germinated seeds were observed by $40 \times$ microscope (Olympus) at $1 d, 2 d, 3 d, 5 d, 6 d, 8 d, 9 d, 11 d$ after incubated. The non-germinated seeds were tested the viability by bromothymol blue and the dead seeds were excluded from calculation. The germination curve was built based on the percentage of germination. Each sample had five dishes (30 seeds per dish) as replications.

$0.1 \mathrm{~g}$ sterile seeds from each sample were collected after incubated for $0 \mathrm{~d}, 4 \mathrm{~d}, 6 \mathrm{~d}$ and $8 \mathrm{~d}$, immediately frozen with liquid nitrogen and stored at $-80^{\circ} \mathrm{C}$. All the samples were homogenized in $1 \mathrm{ml}$ cold distilled water and centrifuged at 12,000 rpm for $5 \mathrm{~min}$ and then collected the supernatants for measurement. The soluble protein content was measured as Bradford methods [53]. The soluble sugar concentration was determined by anthrone method [54]. Three replicates were analyzed for each treatment. 


\section{Effect of exogenous hormones on seed germination of AS and AR}

The peeled seeds of LS and LR were surface sterilized and placed on the petri dishes with one layer of filter paper that moistened with $5 \mathrm{ml}$ waters or tested solutions, including $100 \mu \mathrm{M}$ gibberellin $_{4+7}(\mathrm{GA}), 1 \mu \mathrm{M}$ abscisic acid (ABA), $100 \mu \mathrm{M}$ fluridone $(\mathrm{FL})$ and $1 \mu \mathrm{M}$ paclobutrazol (PA). The seeds were incubated under the same condition as mentioned above. The maximum germination (gMAX) was calculated the number of the germinated seeds divided with the total number of the viable seeds. Each treatment had five dishes (30 seeds per dish) as replications.

\section{RNA isolation and transcriptome sequencing}

The peeled seeds of LS and LR were incubated for $3 d$ and $6 d$ and collected for RNA extraction. Each sample had three biological replications named as LS_3d_1, LS_3d_2, LS_3d_3, LR_3d_1, LR_3d_2, LR_3d_3, LS_6d_1, LS_6d_2, LS_6d_3, LR_6d_1, LR_6d_2, LR_6d_3. RNA extraction and RNA-seq was performed by Novogene Corporation (Beijing, China). RNA degradation and contamination was monitored on $1 \%$ agarose gels. RNA purity was checked using the NanoPhotometer ${ }^{\circledR}$ spectrophotometer (IMPLEN, CA, USA). RNA integrity was assessed using the RNA Nano 6000 Assay Kit of the Agilent Bioanalyzer 2100 system (Agilent Technologies, CA, USA). A total amount of $1.5 \mu \mathrm{g}$ RNA per sample was used as input material for the RNA sample preparations. Sequencing libraries were generated using NEBNext ${ }^{\circledR}$ Ultra $^{\text {TM }}$ RNA Library Prep Kit for Illumina ${ }^{\circledR}$ (NEB, USA) following manufacturer's recommendations.

\section{Transcriptome assembly and functional classification}

Raw reads of fastq format were firstly processed through in-house perl scripts. In this step, clean reads were obtained by removing reads containing adapter, reads containing ploy- $\mathrm{N}$ and low quality reads from raw data. At the same time, Q20, Q30, GC-content and sequence duplication level of the clean data were calculated. All the downstream analysis were based on clean data with high quality. All clean reads of the twelve libraries were obtained using Trinity software to assemble the full-length transcript sequences [55]. Gene function was annotated based on the following databases: NR (NCBI non-redundant protein sequences) $\mathbb{N} \mathrm{NT}$ (NCBI non-redundant nucleotide sequences) $₫ \mathrm{Pfam}$ (Protein family) $\varangle \mathrm{KOG} / \mathrm{COG}$ (Clusters of Orthologous Groups of proteins) $₫$ Swiss-Prot (A manually annotated and reviewed protein sequence

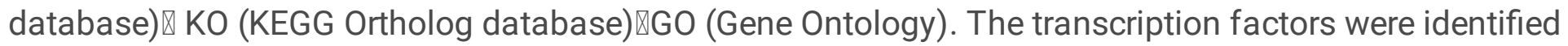
by iTAK program [56].

\section{Differential gene expression and enrichment analysis}

Gene expression levels were calculated using the fragments per kilobase per million fragments (FPKM) method [57]. Differential expression analysis was conducted at $6 \mathrm{~d}$ compared to $3 \mathrm{~d}$ in LS and LR using the DEGseq R package [58]. Q-value $<0.05$ and | log2 (fold change) $\mid>1$ as the threshold for significantly differential expression. All the DEGs were enriched to GO and KEGG database by GOseq and KOBAS software $[59,60]$. The significant enriched GO terms and KEGG pathways were filtered by padj values < 0.05 . 


\section{Quantitative real-time PCR validation}

The quantitative real-time PCR (qRT-PCR) was performed to verify the DEGs identified from the transcriptome results. Total RNA was extracted from the LS and LR seeds under the same condition as the RNA-Seq samples using RNAprep Pure Plant Extraction Kit (Tiangen, China). Then, reverse transcription was performed with $1 \mu \mathrm{g}$ of each RNA sample using CDNA Synthesis kit (TAKARA, China). qRT-PCR was performed with iTaq Universal SYBR Green Supermix (Bio-Rad, USA) on QuantStudio ${ }^{\text {TM }} 1$ real-time PCR System and with the following thermal cycle conditions: denaturing at $95^{\circ} \mathrm{C}$ for $30 \mathrm{~s}$ followed by 40 cycles of $95^{\circ} \mathrm{C}$ for $5 \mathrm{~s}$ and $60^{\circ} \mathrm{C}$ for $1 \mathrm{~min}$. The RT-PCR primers were designed based on the coding sequences (CDS) of the tested genes (Additional file 1. Table S1). UBQ was used as the housekeeping genes. Each treatment had three biological replications and the average values from three technical replications were used for calculating the relative expression level by $2^{-\Delta \Delta C t}$ methods.

\section{Abbreviations}

ACCase

acetyl-CoA carboxylase

DEGs

differentially expressed genes

P.fugax

Polypogon fugax

$\mathrm{GA}$

gibberellin

PA

paclobutrazol

$\mathrm{ABA}$

abscisic acid

$\mathrm{FL}$

fluridone

gMAX

maximum germination

DAI

day after incubated

TCA

tricarboxylic acid

NR

NCBI non-redundant protein sequences

NT

$\mathrm{NCBI}$ non-redundant nucleotide sequences 
Pfam

Protein family

KOG

Clusters of Orthologous Groups of proteins

Swiss-Prot

A manually annotated and reviewed protein sequence database

KEGG

Kyoto Encyclopedia of Genes and Genomes

FPKM

fragments per kilobase per million fragments

qRT-PCR

quantitative real-time PCR

\section{Declarations}

\section{Ethics approval and consent to participate}

There were no specific permits required for plant collection in the selected locations. The sampling locations are not privately owned or natural protected areas. The plants used for the experiments are not considered an endangered or protected species. The collection is legal in China.

\section{Consent for publication}

Not applicable.

\section{Availability of data and materials.}

The raw reads have been deposited in the NCBI Sequence Read Archive (SRA) database (BioProject: PRJNA625626).

\section{Competing interests}

The authors declare that they have no competing interests.

\section{Funding}

This work was financially supported by National Natural Science Foundation of China (No. 31800344). The funder had no role in the design of the study and collection, analysis, and interpretation of data and in writing the manuscript.

\section{Authors' contribution}

$X Y$ contributes to the experiment design and writing; W T contribute to the plant materials preparation; $Y$ $\mathrm{Y}$ and $\mathrm{J} \mathrm{Z}$ contribute to data analysis and revise the paper; $\mathrm{Y} L$ contributes to supervise the experiments 
and revise the paper. All authors reviewed the results and approved the final version of the manuscript.

\section{Acknowledgement}

Not applicable.

\section{References}

1. Casale FA, Giacomini DA, Tranel PJ. Empirical investigation of mutation rate for herbicide resistance. Weed Sci. 2019;67:1-8.

2. Busi R, Gaines TA, Walsh MJ, Powles SB. Understanding the potential for resistance evolution to the new herbicide pyroxasulfone: Field selection at high doses versus recurrent selection at low doses. Weed Res. 2012;52:489-99.

3. Neve P, Powles S. High survival frequencies at low herbicide use rates in populations of Lolium rigidum result in rapid evolution of herbicide resistance. Heredity. 2005;95:485-92.

4. Maxwell BD, Mortimer AM. Selection for Herbicide Resistance. In Herbicide Resistance in Plants: Biology and Biochemistry; Powles, S.B., Holtum, J.A.M., Eds.; Lewis: Boca Raton, FL, USA, 1994; 1 25.

5. Délye C, Michel S. Universal primers for PCR-sequencing of grass chloroplastic acetyl-CoA carboxylase domains involved in resistance to herbicides. Weed Res. 2005;45:323-30.

6. Yu Q, Cairns A, Powles S. Glyphosate, paraquat and ACCase multiple herbicide resistance evolved in a Lolium rigidum biotype. Planta. 2007;225:499-513.

7. Powles SB, Yu Q. Evolution in action: plants resistant to herbicides. Annu Rev Plant Bio. 2010;61:317-47.

8. Cousens RD, Fournier-Level A. Herbicide resistance costs: what are we actually measuring and why? Pest Manag. Sci. 2018;741:539-1546.

9. Vila-Aiub MM, Yu Q, Powles SB. Do plants pay a fitness cost to be resistant to glyphosate? New Phytol. 2019;223:532-47.

10. Anthimidou E, Ntoanidou S, Madesis P, Eleftherohorinos I. Mechanisms of Lolium rigidum multiple resistance to ALS- and ACCase-inhibiting herbicides and their impact on plant fitness. Pestic Biochem Physiol. 2019. DOI:10.1016/j.pestbp.2019.12.010.

11. Menchari Y, Chauvel B, Darmency H, et al. Fitness Costs Associated with Three Mutant Acetylcoenzyme a Carboxylase Alleles Endowing Herbicide Resistance in Black-Grass Alopecurus myosuroides. J Appl Eco. 2008;45(3):939-47. doi:10.1111/j.1365-2664.2008.01462.x.

12. Shergill LS, Boutsalis P, Preston C, et al. Fitness costs associated with 1781 and 2041 ACCasemutant alleles conferring resistance to herbicides in Hordeum glaucum Steud. Crop Prot. 2016;87:60-7.

13. Li YH. Weed Flora of China. 1st ed. Beijing: China Agriculture Press; 1998. p..p 1313-5. [In Chinese]. 
14. Xu ZH, Qi HY, Lu YL, Yang WD, Xie GX. Weed Identification and Management. 1st edn. Hangzhou: Zhejiang University 2014; Press 372 p [in Chinese].

15. Wang K, Qiang Q. Quantitative analysis of distribution of weed communities in wheat fields in the south of Jiangsu province. Journal of Biomathematics. 2005. [in Chinese].

16. Tang W, Zhou F, Chen J, Zhou X. Resistance to ACCase-inhibiting Herbicides in an Asia Minor Bluegrass (Polypogon fugax) Population in China. Pestic Biochem Physiol. 2014;108:16-20.

17. Tang W, Xu X, Shen G, et al. Effect of Environmental Factors on Germination and Emergence of Aryloxyphenoxy Propanoate Herbicide-Resistant and -Susceptible Asia Minor Bluegrass (Polypogon fugax). 2015; 63(3): 669-675. doi: 10.1614/WS-D-14-00156.1.

18. Tang W, Chen J, Yu X, Zhang J, Lu Y. Growth, fecundity, and competition between aryloxyphenoxypropionate-resistant and -susceptible Asia Minor bluegrass (Polypogon fugax). Weed Sci. 2019; 67(5):546-51. doi: 10.1017/ wsc.2019.36.

19. Zhou FY, Zhang Y, Tang W, et al. Transcriptomics analysis of the flowering regulatory genes involved in the herbicide resistance of asia minor bluegrass (Polypogon fugax). BMC Genom. 2017;18:953.

20. Yu Q, Han H, Vila-Aiub MM, Powles SB. AHAS herbicide resistance endowing mutations: Effect on AHAS functionality and plant growth. J Exp Bot. 2010;61:3925-34.

21. Ashigh J, Tardif F. An Ala205Val substitution in acetohydroxyacid synthase of Eastern black nightshade (Solanum ptychanthum) reduces sensitivity to herbicides and feedback inhibition. Weed Sci. 2007;55:558-65.

22. Purrington CB, Bergelson J. Exploring the physiological basis of costs of herbicide resistance in Arabidopsis thaliana. Am Nat. 1999;154:82-91.

23. Paris M, Roux F, Berard A, Reboud X. The effects of the genetic background on herbicide resistance fitness cost and its associated dominance in Arabidopsis thaliana. Heredity. 2008;101:499-506.

24. Frenkel E, Matzrafi M, Rubin B, Peleg Z. Effects of environmental conditions on the fitness penalty in herbicide resistant Brachypodium hybridum. Front Plant Sci. 2017;8:1-10.

25. Williams MMI, Jordan N, Yerkes $\mathrm{C}$. The fitness cost of triazine resistance in jimsonweed (Datura stramonium L.). Am Midl Nat. 1995;133:131-7.

26. Han Z, Wang B, Tian L, Wang S, Zhang J, Guo S, Zhang H, Xu L, Chen Y. Comprehensive dynamic transcriptome analysis at two seed germination stages in maize (Zea mays L.). Physiol Plantarum. 2020; 168(1).

27. Li X, Liu S, Yuan G, et al. Comparative transcriptome analysis provides insights into the distinct germination in sheepgrass (Leymus chinensis) during seed development. Plant Physiol Bioc. 2019; 139.

28. Han C, Yang P. Studies on the molecular mechanisms of seed germination. Proteomics. 2015;15(10):1671-9.

29. Dal Degan F, Rocher A, Cameron-Mills V, von Wettstein D. The expression of serine carboxypeptidases during maturation and germination of the barley grain. P Natl Acad Sci USA. 1994; 91(17). 
30. Shu K, Liu X, Xie Q, He Z. Two Faces of One Seed: Hormonal Regulation of Dormancy and Germination. Mol Plant. 2016;9:34-45.

31. Goggin DE, Steadman KJ, Emery RJ, Farrow SC, Benech-Arnold RL, Powles SB. ABA inhibits germination but not dormancy release in mature imbibed seeds of Lolium rigidum Gaud. J Exp Bot. 2009;60(12):3387-96.

32. Sun TP, Kamiya Y. The Arabidopsis GA1 locus encodes the cyclase ent-kaurene synthetase A of gibberellin biosynthesis. Plant Cell. 1994;6(10):1509-18. doi:10.1105/tpc.6.10.1509.

33. Lee S, Cheng H, King KE, Wang W, Peng J. Gibberellin regulates Arabidopsis seed germination via rgl2, a GAl/RGA-like gene whose expression is up-regulated following imbibition. Gene Dev. 2002;16(5):646-58.

34. Yamaguchi S, Smith MW, Brown Robert GS, Kamiya Y, Sun T. Phytochrome regulation and differential expression of gibberellin 3 $\beta$-hydroxylase genes in germinating Arabidopsis seeds. Plant Cell. 1998;10(12):2115-26.

35. de Lucas M, Davière JM, Rodríguez-Falcón M, Pontin M, Iglesias-Pedraz JM, Lorrain S, Fankhauser C, Blázquez MA, Titarenko E, Prat S. A molecular framework for light and gibberellin control of cell elongation. Nature. 2008;451(7177):480-4.

36. Lefebvre V, North H, Frey A, Sotta B, Seo M, Okamoto M, Nambara E, Marion-Poll A. Functional analysis of Arabidopsis NCED6 and NCED9 genes indicates that ABA synthesized in the endosperm is involved in the induction of seed dormancy. The Plant journal. 2006;45(3):309-19.

37. Lindgren LO, Stalberg KG, Hoglund AS. Seed-specific overexpression of an endogenous Arabidopsis phytoene synthase gene results in delayed germination and increased levels of carotenoids, chlorophyll, and abscisic acid. Plant physiol. 2003;132(2):779-85.

38. Chae MJ, Lee JS, Nam MH, Cho K, Hong JY, Yi SA, Suh SC, Yoon IS. A rice dehydration-inducible SNF1-related protein kinase 2 phosphorylates an abscisic acid responsive element-binding factor and associates with ABA signaling. Plant Mol Biol. 2007;63(2):151-69.

39. Fujii H, Zhu JK. Arabidopsis mutant deficient in 3 abscisic acid-activated protein kinases reveals critical roles in growth, reproduction, and stress. Proc Natl Acad Sci USA. 2009;106(20):8380-5.

40. Brown AP, Slabas AR, Rafferty JB. Fatty Acid Biosynthesis in Plants Metabolic Pathways, Structure and Organization. In: Wada H, Murata N, editors. Lipids in Photosynthesis. Advances in Photosynthesis and Respiration. Vol. 30. Dordrecht: Springer; 2009.

41. Ohlrogge J, Browse J. Lipid Biosynthesis. Plant Cell. 1995;7:957-70.

42. Nikolau BJ, Ohlrogge JB, Wurtele ES. Plant biotin-containing carboxylases. Arch Biochem Biophys. 2003;414:211-22.

43. [U31]Zhang H, Tweel B, Tong L. Molecular basis for the inhibition of the carboxytransferase domain of acetyl-coenzyme-A carboxylase by haloxyfop and diclofop. Proc Natl Acad Sci USA. 2004;101:5910-5.

44. Vila-Aiu MM, Neve P, Steadman KJ, Powles SB. Ecological fitness of a multiple herbicide-resistant Lolium rigidum population: Dynamics of seed germination and seedling emergence of resistant and 
susceptible phenotypes. J Appl Ecol. 2005;42:288-98.

45. Délye C, Menchari Y, Michel S, Cadet E, Le Corre V. A new insight into arable weed adaptive evolution: Mutations endowing herbicide resistance also affect germination dynamics and seedling emergence. Ann Bot. 2013;111:681-91.

46. Owen MJ, Michael PJ, Renton M, Steadman KJ, Powles SB. Towards large-scale prediction of Lolium rigidum emergence. II. Correlation between dormancy and herbicide resistance levels suggests an impact of cropping systems. Weed Res. 2011;51:133-41.

47. Kim ST, Wang Y, Kang SY, Kim SG, Rakwal R, Kim YC, Kang KY. Developing rice embryo proteomics reveals essential role for embryonic proteins in regulation of seed germination. J Proteome Res. 2009;8(7):3598-605.

48. Rylott EL. The gluconeogenic enzyme phosphoenolpyruvate carboxykinase in Arabidopsis is essential for seedling establishment. Plant Physiol. 2009;131(4):1834-42.

49. Kim ST, Wang Y, Kang SY, Kim SG, Rakwal R, Kim YC, Kang KY. Developing rice embryo proteomics reveals essential role for embryonic proteins in regulation of seed germination. J Proteome Res. 2009;8(7):3598-605.

50. Sew YS, Stroher E, Fenske R, Millar AH. Loss of mitochondrial malate dehydrogenase activity alters seed metabolism impairing seed maturation and post-germination growth in Arabidopsis. Plant Physiol. 2016;171(2):849-63. doi:10.1104/pp.16.01654.

51. Pracharoenwattana I, Smith CSM. Arabidopsis peroxisomal citrate synthase is required for fatty acid respiration and seed germination. Plant Cell. 2005;17(7):2037-48.

52. Muscolo A, Panuccio MR, Sidari M. (2001). The effect of phenols on respiratory enzymes in seed germination. Plant Growth Regul. 2001; 35(1): 31-35.

53. Bradford MM. A rapid method for the quantitation of microgram quantities of protein utilizing the principle of protein-dye binding. Anal Biochem. 1976;72:248-54.

54. Hansen J, Møller I. Percolation of starch and soluble carbohydrates from plant tissue for quantitative determination with anthrone. Anal Biochem. 1975;68:87-94.

55. Grabherr MG, Haas BJ, Yassour M, et al. Full-length transcriptome assembly from RNA-Seq data without a reference genome. Nat Biotechnol. 2011;29:644-52.

56. Zheng Y, Jiao C, Sun H, Rosli HG, Pombo MA, Zhang P, Banf M, Dai X, Martin GB, Giovannoni JJ, Zhao PX, Rhee SY. Fei Z iTAK: a program for genome-wide prediction and classification of plant transcription factors, transcriptional regulators, and protein kinases. Mol Plant. 2016;9:1667-70.

57. Trapnell C, Williams BA, Pertea G, et al. Transcript assembly and quantification by RNA-Seq reveals unannotated transcripts and isoform switching during cell differentiation. Nat Biotech. 2010;28:5115.

58. Love MI, Huber W, Anders S. Moderated estimation of fold change and dispersion for RNA-seq data with DESeq2. Genome Biol. 2014;15:550. doi:10.1186/s13059-014-0550-8. 
59. Young MD, Wakefield MJ, Smyth GK, et al. Gene ontology analysis for RNA-seq: accounting for selection bias. Genome Biol. 2010. doi:10.1186/gb-2010-11-2-r14.

60. Mao X, Cai T, Olyarchuk JG, et al. Automated genome annotation and pathway identification using the KEGG Orthology (KO) as a controlled vocabulary. Bioinformatics. 2005;21:3787-93.

\section{Tables}

Table 1 Overview of the sequencing reads obtained from each sample.

\begin{tabular}{llllllll}
\hline Sample & Raw Reads & Clean Reads & Clean Bases & Error (\%) & Q20 (\%) & Q30 (\%) & GC Content (\%) \\
\hline LR_3d_1 & 46947070 & 46112196 & $6.92 \mathrm{G}$ & 0.03 & 97.95 & 94.2 & 55.51 \\
LR_3d_2 & 57819080 & 56883382 & $8.53 \mathrm{G}$ & 0.03 & 97.89 & 94.03 & 55.32 \\
\hline LR_3d_3 & 61713628 & 60389070 & $9.06 \mathrm{G}$ & 0.03 & 97.87 & 93.97 & 55.07 \\
\hline LR_6d_1 & 55934224 & 55303162 & $8.3 \mathrm{G}$ & 0.02 & 98 & 94.24 & 55.02 \\
\hline LR_6d_2 & 53631602 & 52984034 & $7.95 \mathrm{G}$ & 0.02 & 98.03 & 94.3 & 55.09 \\
\hline LR_6d_3 & 63912876 & 63221906 & $9.48 \mathrm{G}$ & 0.03 & 97.92 & 94.08 & 55.1 \\
\hline LS_3d_1 & 56261812 & 55216600 & $8.28 \mathrm{G}$ & 0.03 & 97.83 & 93.9 & 54.98 \\
\hline LS_3d_2 & 56163918 & 55050700 & $8.26 \mathrm{G}$ & 0.03 & 97.84 & 93.88 & 54.59 \\
\hline LS_3d_3 & 56540874 & 55577868 & $8.34 \mathrm{G}$ & 0.03 & 97.89 & 94.03 & 54.98 \\
\hline LS_6d_1 & 57849026 & 56878644 & $8.53 \mathrm{G}$ & 0.03 & 97.88 & 93.98 & 54.88 \\
\hline LS_6d_2 & 48963372 & 48180318 & $7.23 \mathrm{G}$ & 0.03 & 97.92 & 94.1 & 54.63 \\
\hline LS_6d_3 & 49585538 & 48901900 & $7.34 \mathrm{G}$ & 0.02 & 98.01 & 94.28 & 53.51 \\
\hline
\end{tabular}

Table 2 Functional annotations of unigenes in the databases 


\begin{tabular}{lcc}
\hline & Number of Unigenes & Percentage (\%) \\
\hline Annotated in NR & 67183 & 44.98 \\
Annotated in NT & 68540 & 45.89 \\
\hline Annotated in KO & 22834 & 15.29 \\
\hline Annotated in SwissProt & 52642 & 35.25 \\
\hline Annotated in PFAM & 61384 & 41.1 \\
\hline Annotated in GO & 61384 & 41.1 \\
\hline Annotated in KOG & 20042 & 13.42 \\
\hline Annotated in all Databases & 8625 & 5.77 \\
\hline Annotated in at least one Database & 99209 & 66.43 \\
\hline Total Unigenes & 149330 & 100 \\
\hline
\end{tabular}

Table 3 DEGs significantly up- or down- regulated in LS and LR comparison settings. 


\begin{tabular}{|c|c|c|c|c|}
\hline Gene ID & $\begin{array}{c}\log _{2} \mathrm{FC} \\
\left(\mathrm{LS} \_6 \mathrm{~d} \text { vs LS_3d) }\right.\end{array}$ & $\begin{array}{c}\log _{2} \mathrm{FC} \\
\text { (LR_6d vs LR_3d) }\end{array}$ & Encoded protein & Description \\
\hline \multicolumn{5}{|c|}{ Carbohydrate metabolism } \\
\hline Cluster-37472.6545 & 7.36 & 3.84 & GPI & glucose-6-phosphate isomerase \\
\hline Cluster-37472.71503 & 5.65 & 2.84 & FBA & fructose-bisphosphate aldolase \\
\hline Cluster-40718.0 & 5.96 & 4.88 & PDHA & pyruvate dehydrogenase E1 component alpha subunit \\
\hline Cluster-35171.2 & 6.98 & 5.61 & PCKA & phosphoenolpyruvate carboxykinase (ATP) \\
\hline Cluster-37472.39187 & 3.80 & 1.85 & $\mathrm{ADH}$ & alcohol dehydrogenase class-P \\
\hline Cluster-37472.47005 & 4.19 & 2.04 & $\mathrm{ADH}$ & alcohol dehydrogenase class-P \\
\hline Cluster-37472.85108 & 8.43 & 6.07 & $\mathrm{MDH}$ & malate dehydrogenase \\
\hline Cluster-15133.3 & 6.95 & 3.19 & $\mathrm{MDH}$ & malate dehydrogenase \\
\hline Cluster-37472.21027 & 9.41 & 4.35 & CS & citrate synthase \\
\hline Cluster-33485.0 & 6.88 & 5.55 & OGDH & 2-oxoglutarate dehydrogenase E1 component \\
\hline Cluster-38121.0 & 7.15 & 5.66 & LSC1 & succinyl-CoA synthetase alpha subunit \\
\hline Cluster-34010.0 & 5.12 & 3.97 & LSC2 & succinyl-CoA synthetase beta subunit \\
\hline Cluster-37472.82495 & 9.25 & 6.45 & SDHB & succinate dehydrogenase (ubiquinone) iron-sulfur subunit \\
\hline Cluster-39676.0 & 7.51 & 4.56 & SDHB & succinate dehydrogenase (ubiquinone) iron-sulfur subunit \\
\hline Cluster-41938.0 & 7.25 & 4.02 & PYC & pyruvate carboxylase \\
\hline Cluster-37472.72264 & 8.37 & 3.93 & PGD & 6-phosphogluconate dehydrogenase \\
\hline Cluster-37472.83492 & 6.66 & 5.71 & TKT & transketolase \\
\hline Cluster-37472.237 & 7.72 & 4.88 & gntK & gluconokinase \\
\hline
\end{tabular}

\section{Fatty acid metabolism}

\begin{tabular}{lccll}
\hline Cluster-39490.0 & 7.28 & 4.20 & ACACA & acetyl-CoA carboxylase / biotin carboxylase 1 \\
\hline Cluster-31226.0 & 7.10 & 4.82 & FabG & 3-oxoacyl-[acyl-carrier protein] reductase \\
\hline Cluster-37472.4287 & 9.16 & 5.52 & ACSL & long-chain acyl-CoA synthetase \\
\hline Cluster-33548.0 & 9.20 & 5.34 & ACAA1 & acetyl-CoA acyltransferase 1 \\
\hline Cluster-37472.85335 & 9.90 & 5.52 & ACADM & acyl-CoA dehydrogenase \\
\hline Cluster-30044.0 & 7.77 & 4.75 & ACAT & acetyl-CoA C-acetyltransferase
\end{tabular}

\section{Hormones biosynthesis and signal transduction}

\begin{tabular}{lllll}
\hline Cluster-37472.24032 & 9.69 & 3.87 & KS & ent-copalyl diphosphate synthase \\
\hline Cluster-37472.23672 & 6.42 & 4.78 & KS & ent-kaurene synthase \\
\hline Cluster-37472.70387 & 3.31 & 2.02 & GA3ox & gibberellin 3-beta-dioxygenase \\
\hline Cluster-51396.0 & -1.69 & 3.01 & PIF4 & phytochrome-interacting factor 4 \\
\hline Cluster-37472.23110 & -1.97 & 1.81 & NCED & 9-cis-epoxycarotenoid dioxygenase \\
\hline Cluster-37472.23992 & 2.73 & 3.36 & PSY & phytoene synthase \\
\hline Cluster-37472.76697 & 1.64 & 2.53 & LcyE & lycopene epsilon-cyclase
\end{tabular}




\section{Supplementary Information}

Additional file 1: RT-PCR primers

Additional file 2: KOG classification

Additional file 3: GO classification

Additional file 4: KEGG classification

Additional file 5: Go function enrichment of up- or down-regulated DEGs at 3DAl compared with 6 DAl in LS

Additional file 6: Go function enrichment of up- or down-regulated DEGs at 3DAl compared with 6 DAI in LR

Figures 
a
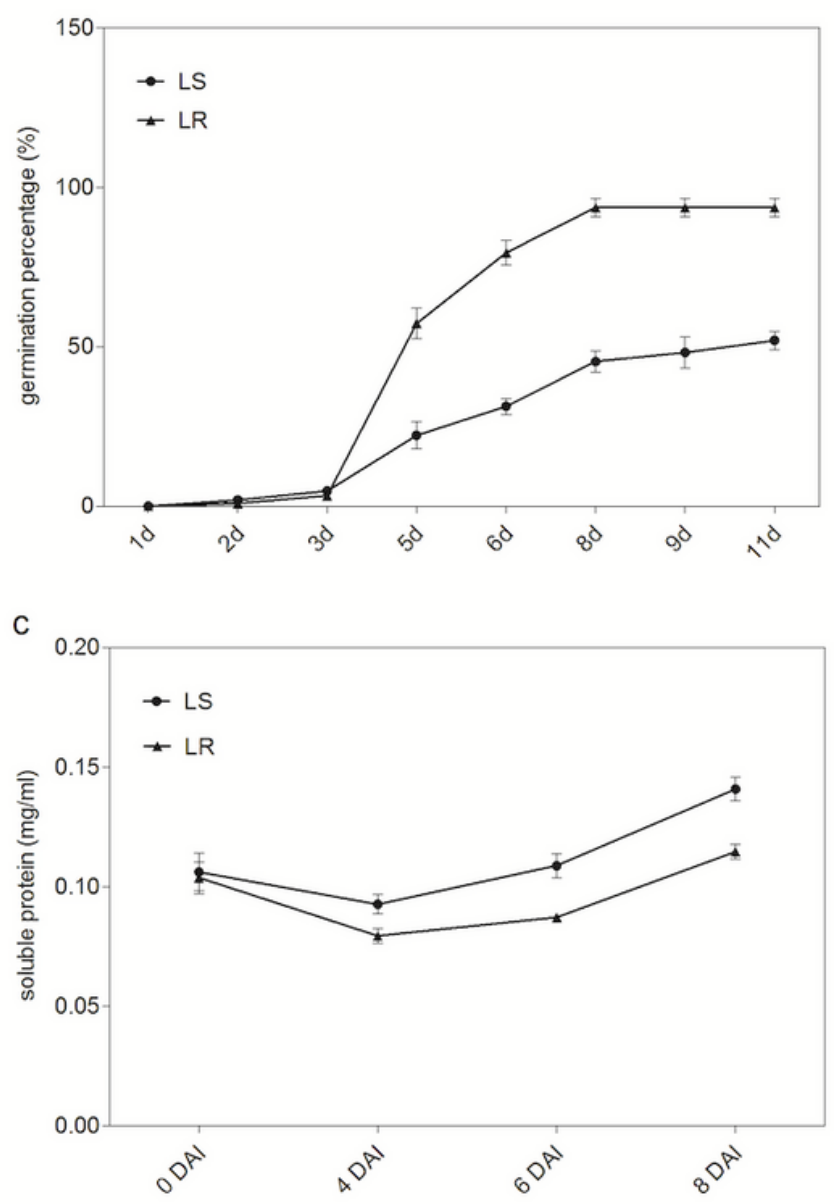

b

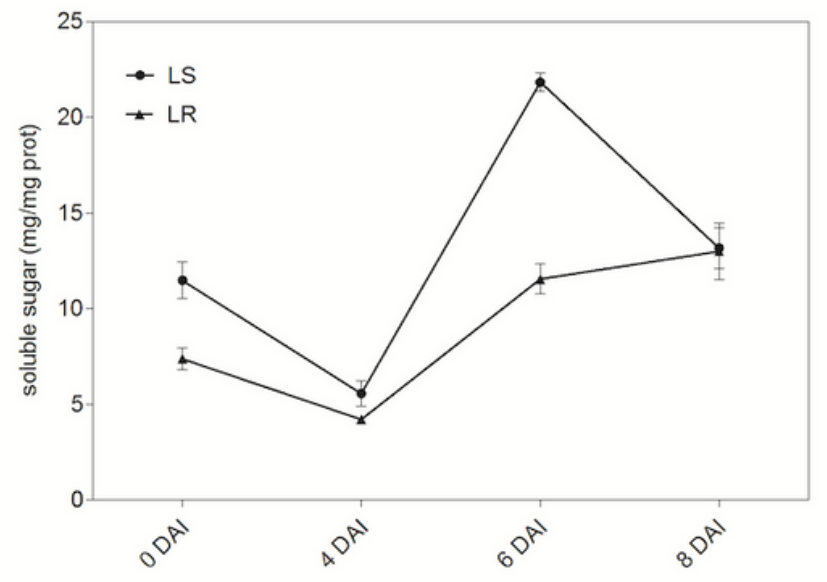

d

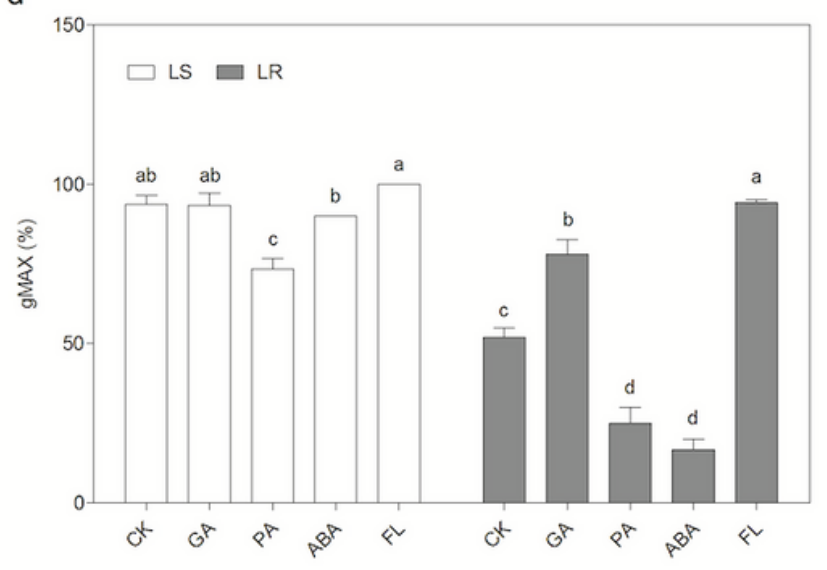

\section{Figure 1}

Seed germination characters in LS and LR. a Dynamic change of germination percentage of LS and LR seeds; $b$ and c Dynamics change of soluble sugar and protein content of LS and LR seeds. $d$. The maximum germination (gMAX) of LS and LR with exogenous hormones application. CK: water, GA: gibberellin, PA: paclobutrazol, ABA: abscisic acid, FL: fluridone. Bars are mean \pm standard error $(n=5)$. Values in a column followed by the different letters means significant differences $(P \leq 0.05)$. 
A

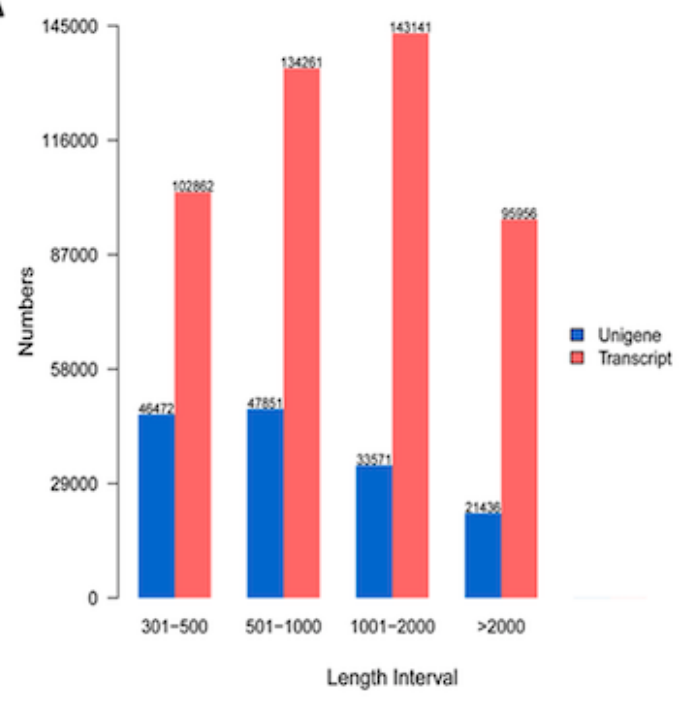

B Species classification

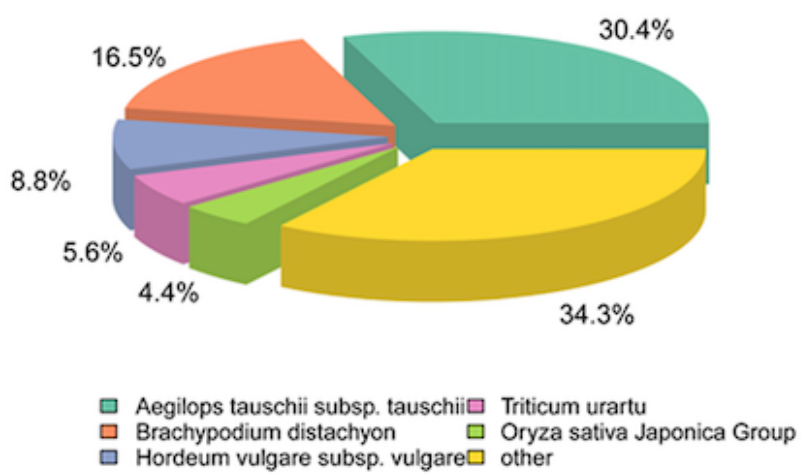

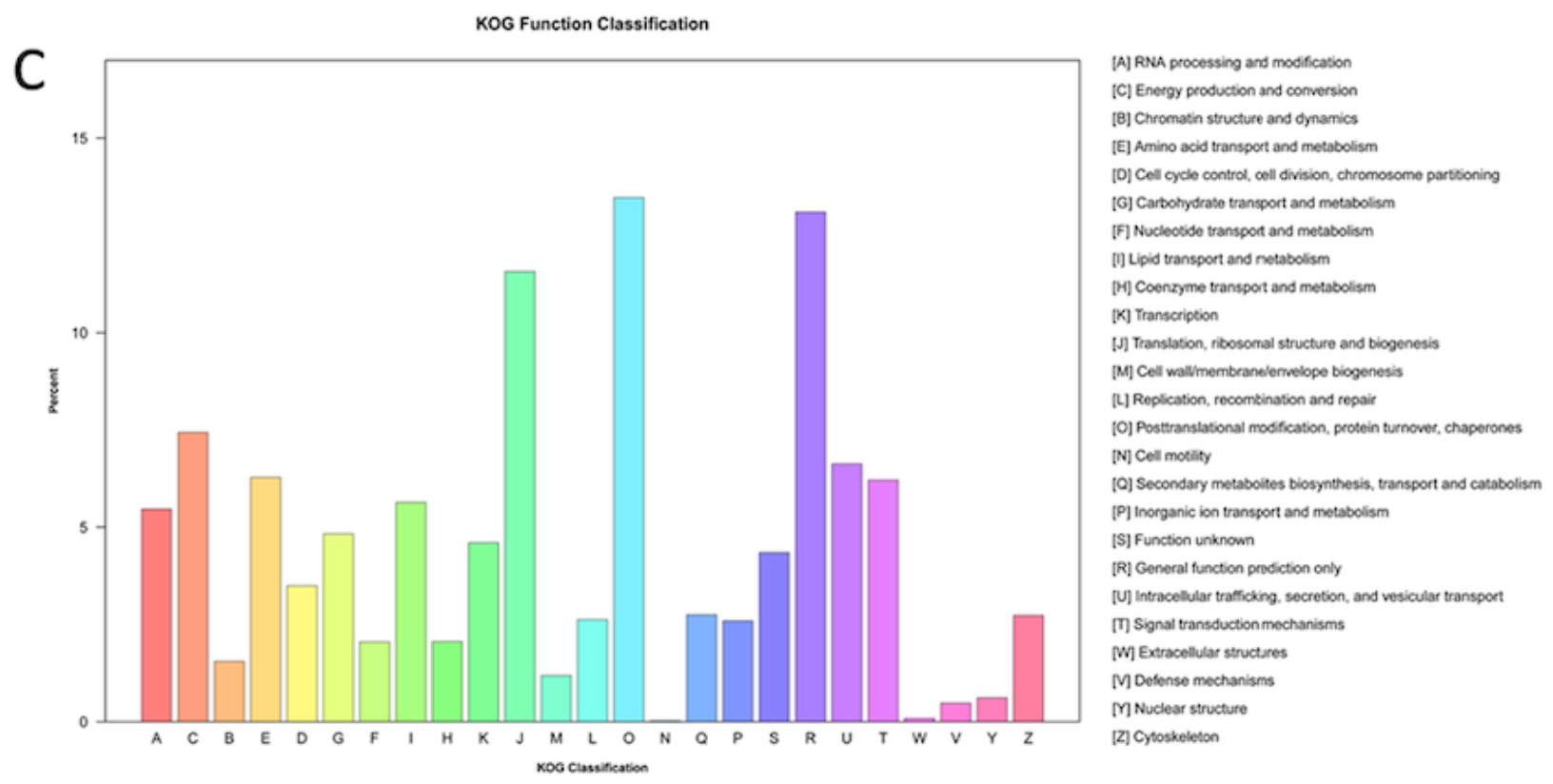

Figure 2

Transcriptome assembly and unigenes annotation of P.fugax. a The length distribution of unigenes and transcripts; $b$ Species classification of P. fugax transcriptome annotated to NR database. The numbers near the pie charts indicate the percentage of unigenes in each class; c KOG function classification of unigenes. 

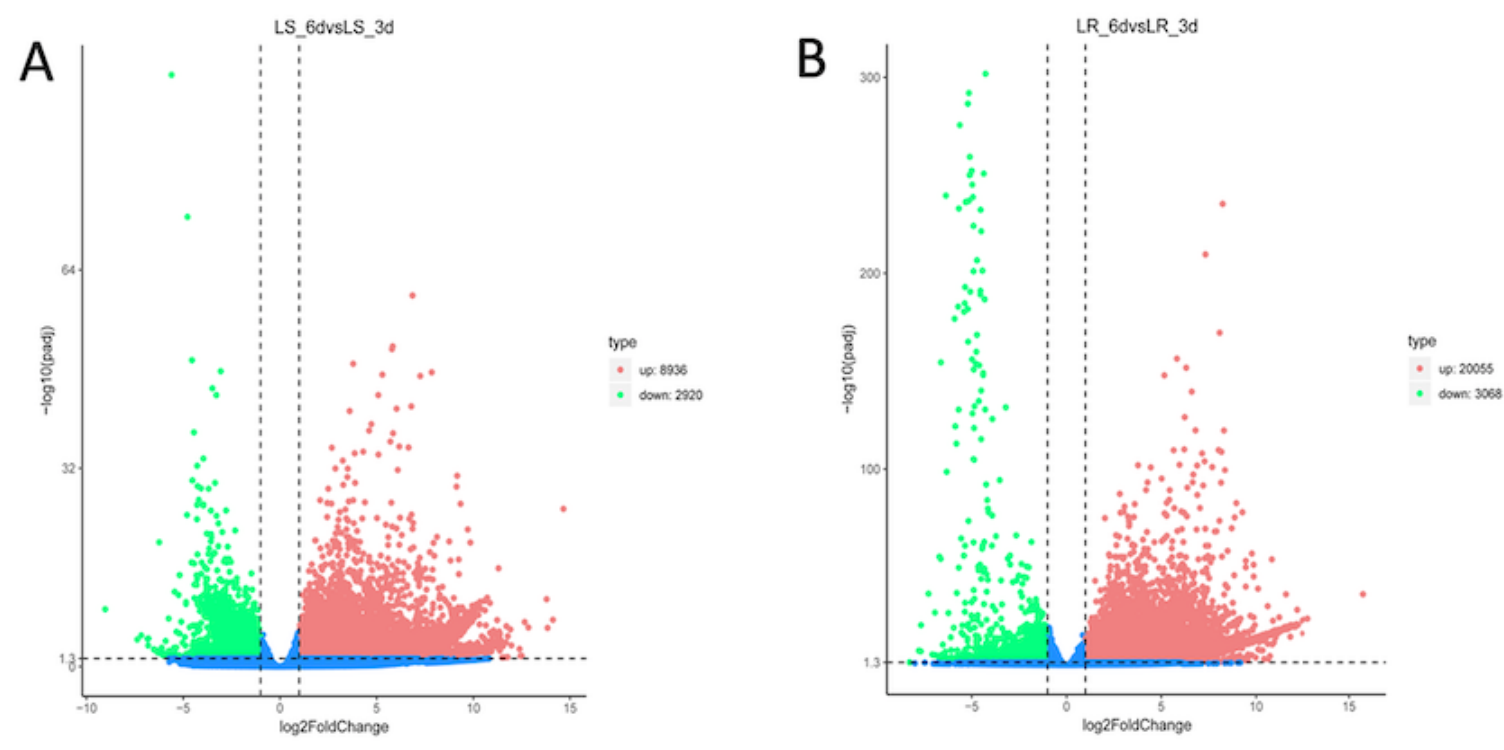

C

Pearson correlation between samples

LR_3d_3 - 0.6090 .5350 .5530 .7120 .6380 .7040 .7510 .7530 .7520 .9470 .894

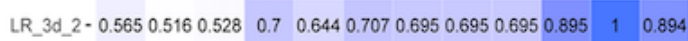

$\begin{array}{llllllllllll}\text { LR_3d_1- } 0.616 & 0.554 & 0.57 & 0.722 & 0.653 & 0.712 & 0.759 & 0.758 & 0.76 & 1 & 0.895 & 0.947\end{array}$

LS_3d_3- $0.7620 .6840 .7150 .57 \quad 0.5210 .5480 .9440 .941 \quad 1 \quad 0.760 .6950 .752$

LS_3d_2- $0.750 .6690 .6970 .5620 .5110 .5430 .943 \quad 1 \quad 0.9410 .7580 .6950 .753$

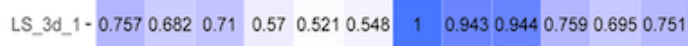

LR_6d_3 - 0.561 $0.6690 .6170 .8740 .875 \quad 1 \quad 0.5480 .5430 .5480 .7120 .7070 .704$

LR_6d_2- $0.5870 .7170 .6470 .874 \quad 1 \quad 0.8750 .5210 .5110 .5210 .6530 .6440 .638$

$\begin{array}{lllllllllllllll}\text { LR_6d_1 } 0.602 & 0.695 & 0.654 & 1 & 0.874 & 0.874 & 0.57 & 0.562 & 0.57 & 0.722 & 0.7 & 0.712\end{array}$

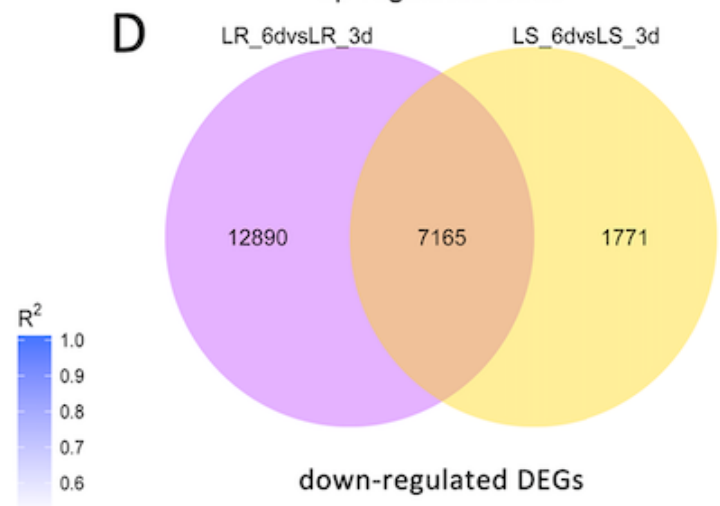

D

up-regulated DEGs

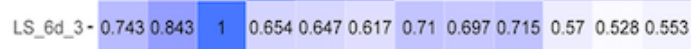

LS_6d_2-0.724 10.8430 .6950 .7170 .6690 .6820 .6690 .6840 .5540 .5160 .535

LS_6d_1- 110.7240 .7430 .6020 .5870 .5610 .7570 .750 .7620 .6160 .5650 .609

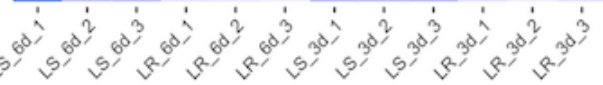

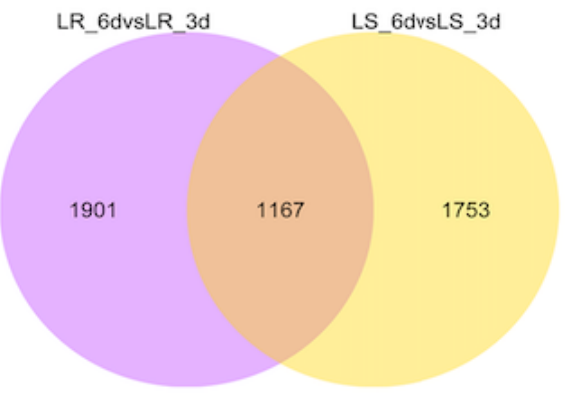

Figure 3

Analysis of different expression genes (DEGs) in LS comparison setting (LS_6d vs LS_3d) and LR comparison setting (LR_6d vs LR_3d). $a, b$ the volcano plot of DEGs in LR and LS. Green dots represent the significant down-regulated DEGs. Red dots represent the significant up-regulated DEGs. c Correlation cluster between three biological replicates of LS_3d, LS_6d, LR_3d and LR_6d; d Venn diagram of upregulated and down-regulated DEGs in LS and LR comparison settings. 

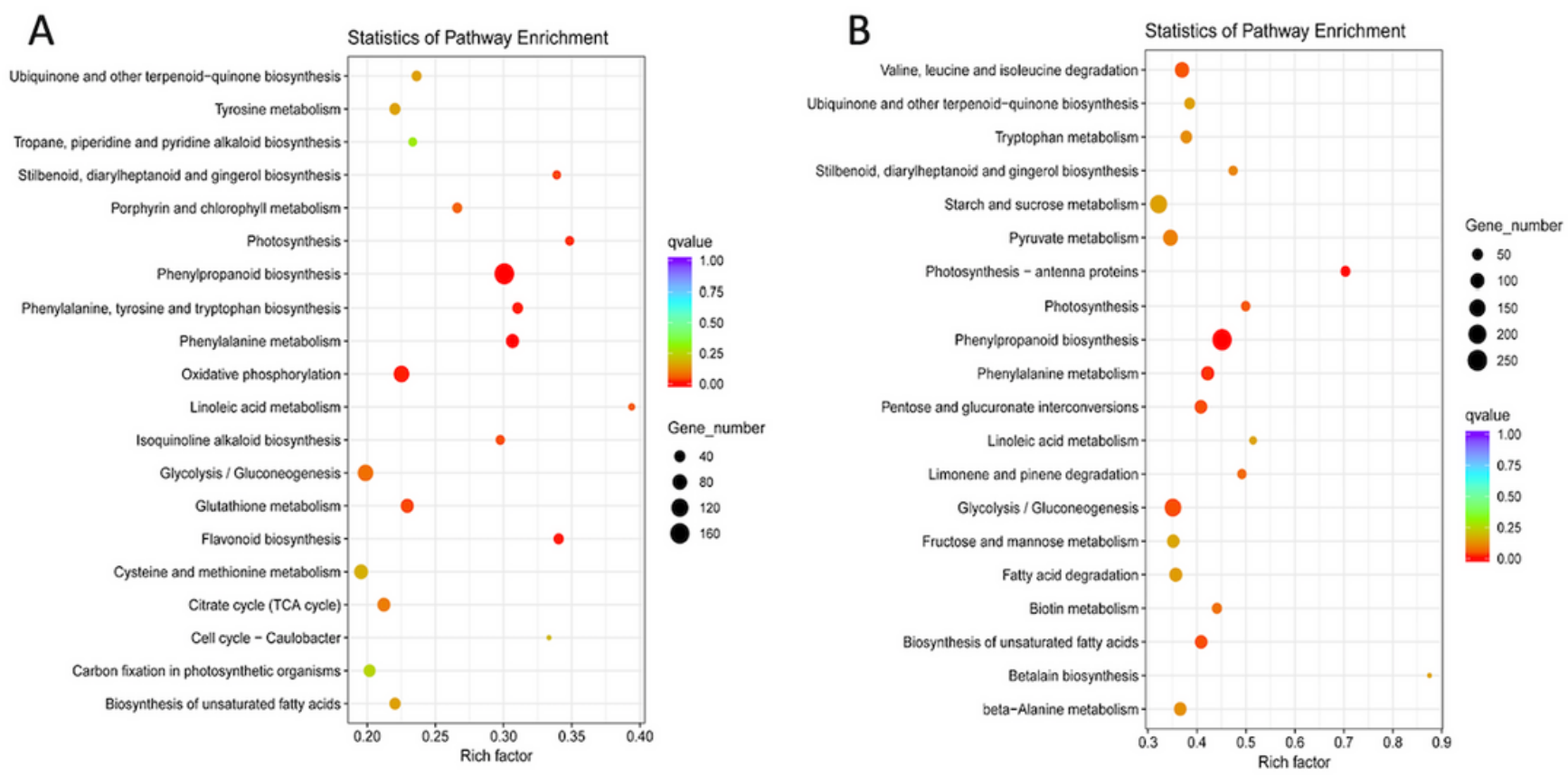

Figure 4

KEGG enrichment analysis of the up-regulated DEGs. a Top 20 enriched pathways in LS comparison setting (LS_6d vs LS_3d); b Top 20 enriched pathways in LR comparison setting (LR_6d vs LR_3d); The colors are shaded according to the q-values level as shown in the color bars; the size of the circle indicates the number of DEGs. 
a

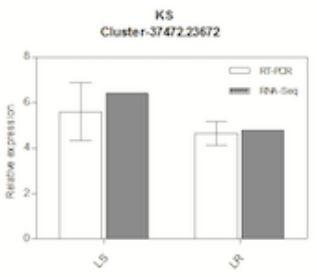

$\underset{\text { chuster-374 }}{\text { crte }}$

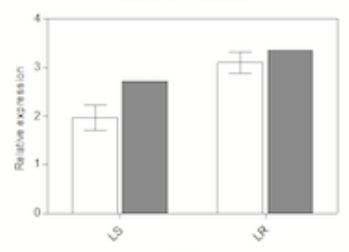

Monz

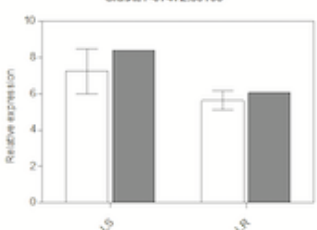

pckA

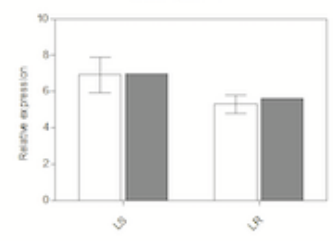

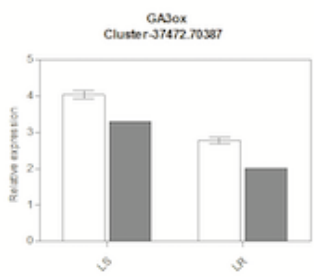

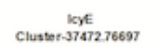

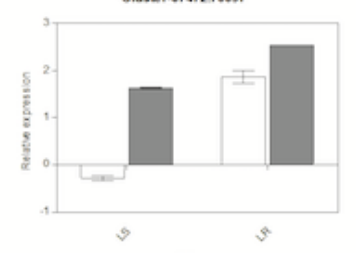

CS

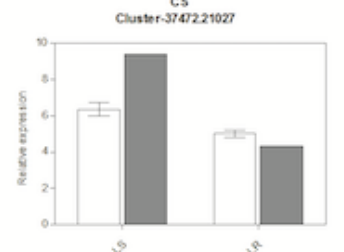

MDH1

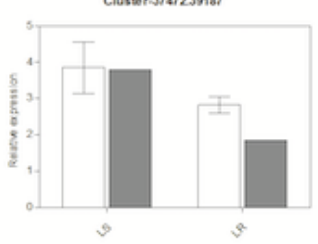

Clutuersismo:

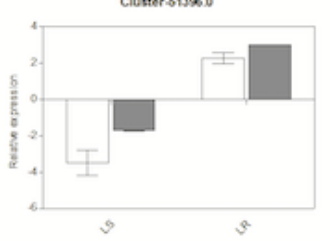

SNRK2
Cluster -374726593

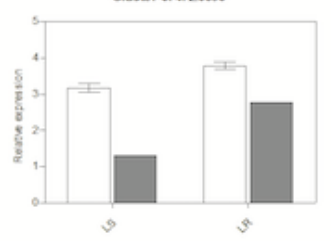

LSC1
Clutters\$1210

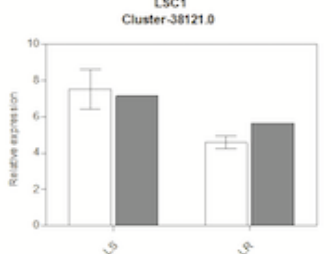

Setuster 3782203482

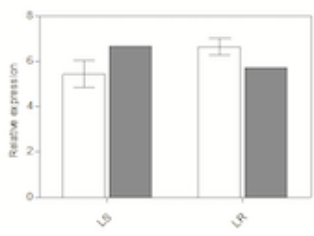

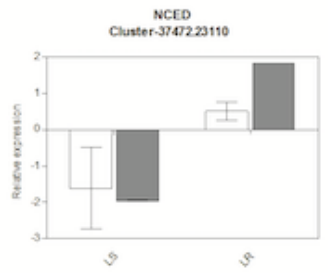

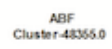

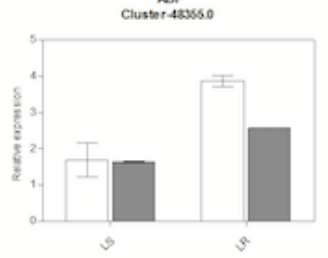

Cluturestinsoso
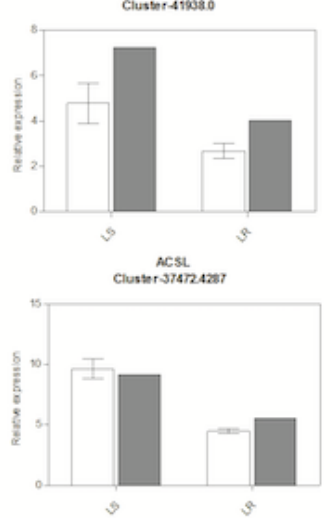

b

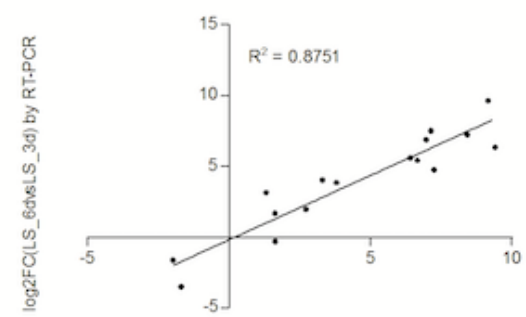

$\log 2$ FC(LS_6dusLS_3d) by RNA-Seq

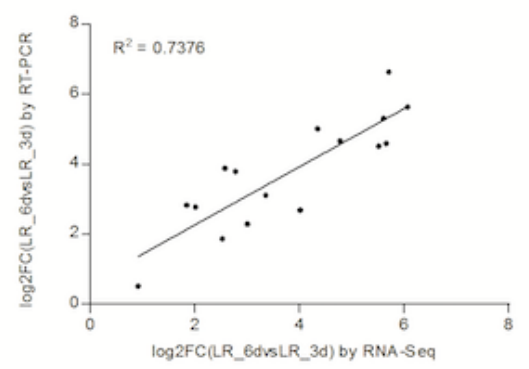

\section{Figure 5}

The validation of RNA-seq results using qRT-PCR. a The relative expression levels of 16 DEGs determine by qRT-PCR (white column) and RNA-Seq (grey column). RNA-Seq results (Fold change) and qRT-PCR results $(2-\Delta \Delta \mathrm{Ct})$ were transformed to log2 forms. Bars are mean \pm standard error $(n=3)$. $b$ Linear regression analysis of the RNA-Seq and qRT-PCR results. The R2 values represent the correlation between RNA-Seq and qRT-PCR results. 


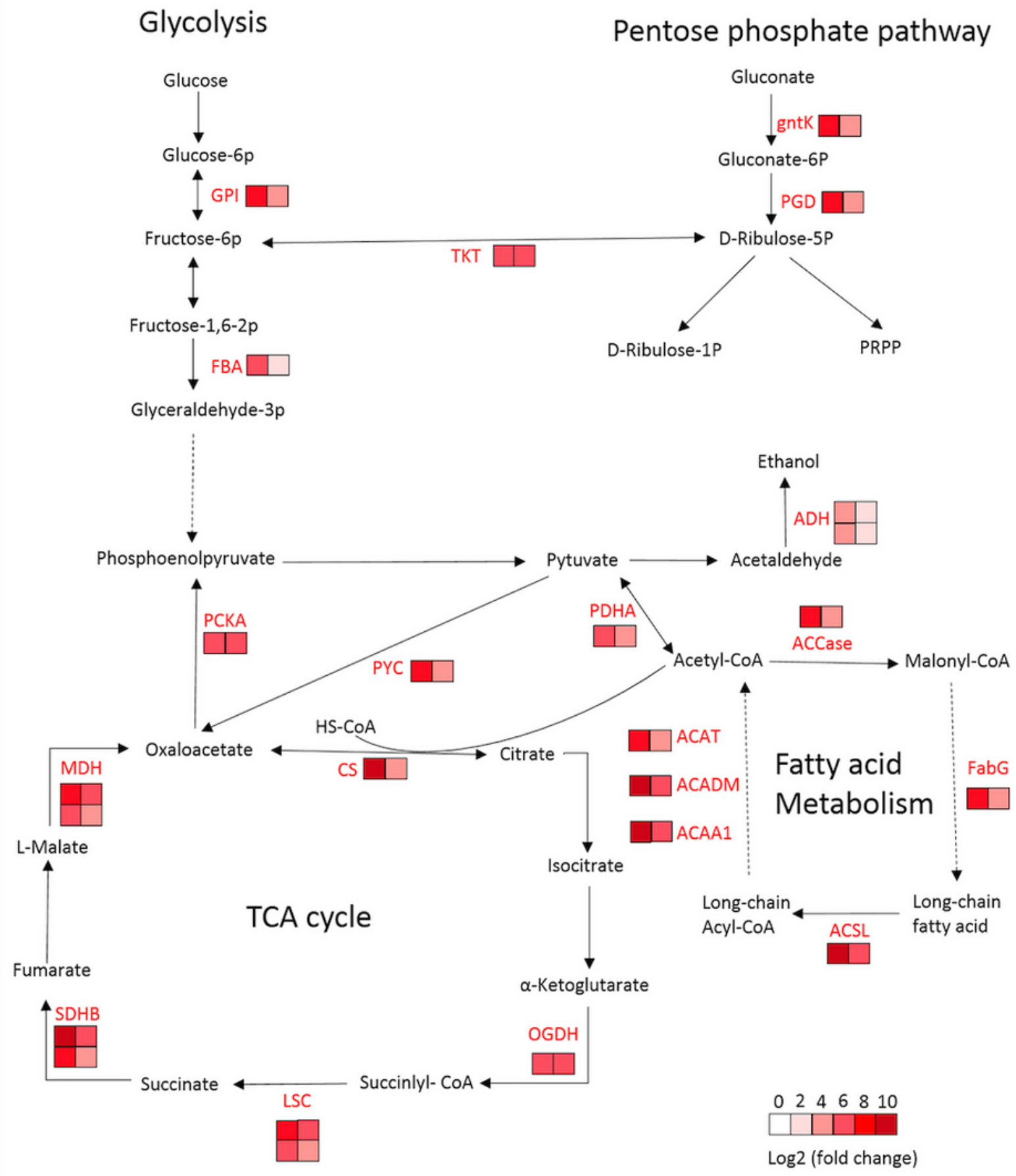

\section{Figure 6}

Visualization of DEGs involved in carbohydrate metabolism and fatty acid metabolism. Substrates are in black font and enzymes in red font. The color of boxes represents Log2 (fold change) values based on the transcriptome results. The values are shown by a color gradient from low (white) to high (red). The left column of boxes represents the Log2 (fold change) value obtained from LS comparison setting, and 
the right column represents that from LR comparison setting. Each row of boxes represents one DEG. Solid arrows represent direct processes, and dashed arrows represent indirect processes.

\section{Supplementary Files}

This is a list of supplementary files associated with this preprint. Click to download.

- Additionalfile1RTPCRprimers.xlsx

- Additionalfile2KOGclassification.xlsx

- Additionalfile3GOclassification.xls

- Additionalfile4KEGGclassification.xls

- Additionalfile5LS6dvsLS3dDEGGOenrichmentsig.xlsx

- Additionalfile6LR6dvsLR3dDEGGOenrichmentsig.xIsx 